\title{
CDP1, a novel component of chloroplast division site positioning system in Arabidopsis
}

\author{
Min Zhang ${ }^{1}$, Yong $\mathrm{Hu}^{1}$, Jingjing Jia ${ }^{1}$, Dapeng $\mathrm{Li}^{1}$, Runjie Zhang ${ }^{1}$, Hongbo Gao ${ }^{2}$, Yikun $\mathrm{He}^{1}$ \\ ${ }^{I}$ College of Life Science, Capital Normal University, Beijing 100048, China; ${ }^{2}$ College of Biological Sciences and Biotechnology, \\ Beijing Forestry University, Beijing 100083, China
}

Chloroplasts are plant-specific organelles that evolved from endosymbiotic cyanobacteria. They divide through binary fission. Selection of the chloroplast division site is pivotal for the symmetric chloroplast division. In $E$. coli, positioning of the division site at the midpoint of the cell is regulated by dynamic oscillation of the Min system, which includes MinC, MinD and MinE. Homologs of MinD and MinE in plants are involved in chloroplast division. The homolog of MinC still has not been identified in higher plants. However, an FtsZ-like protein, ARC3, was found to be involved in chloroplast division site positioning. Here, we report that chloroplast division site positioning 1 (AtCDP1) is a novel chloroplast division protein involved in chloroplast division site placement in Arabidopsis. AtCDP1 was discovered by screening an Arabidopsis cDNA expression library in bacteria for colonies with a cell division phenotype. AtCDP1 is exclusively expressed in young green tissues in Arabidopsis. Elongated chloroplasts with multiple division sites were observed in the loss-of-function $c d p 1$ mutant. Overexpression of AtCDP1 caused a chloroplast division phenotype too. Protein interaction assays suggested that AtCDP1 may mediate the chloroplast division site positioning through the interaction with ARC3. Overall, our results indicate that AtCDP1 is a novel component of the chloroplast division site positioning system, and the working mechanism of this system is different from that of the traditional MinCDE system in prokaryotic cells.

Keywords: Arabidopsis, chloroplast division, AtCDP1

Cell Research (2009) 19:877-886. doi: 10.1038/cr.2009.78; published online 30 June 2009

\section{Introduction}

The prokaryotic origin of chloroplasts in plant cells is widely accepted [1-4]. Many studies on chloroplast division have demonstrated that chloroplasts undergo division by a process of binary fission [5-8]. It is important that the division site is localized at the midpoint of chloroplasts during this process to ensure equal amount of chloroplast components in two daughter chloroplasts and maintain relatively stable number and size of chloroplasts in the cell in higher plants $[2,5,9]$.

In $E$. coli, cell division needs a tubulin-related protein,

Correspondence: Yikun $\mathrm{He}^{\mathrm{a}}$, Hongbo Gao

${ }^{\mathrm{a}}$ Tel: +86-10-68902203; Fax: +86-10-68902203

E-mail: yhe@mail.cnu.edu.cn

${ }^{\mathrm{b}}$ Tel: +86-10-62336496

E-mail: gaohongbo@bjfu.edu.cn

Received 30 May 2009; revised 5 June 2009; accepted 5 June 2009; published online 30 June 2009
FtsZ, to form a ring at the midpoint of the long axis of the cell, which results in the recruitment of many other cell division proteins and the symmetric cell division $[10,11]$. Correct localization of the FtsZ-ring (Z-ring) is mediated by dynamic oscillation of the Min system, which includes three proteins MinC, MinD and MinE [12, 13]. MinC is an inhibitor of cell division. It is activated by MinD, which is a membrane-bound ATPase. The MinCD complex prevents FtsZ polymerization in the cell $[14,15]$. MinE is a topological protein, which forms a dynamic structure to repetitively drive MinCD complex first to one cell pole and then to the opposite pole [16]. Controlled by this precise oscillation of Min proteins, FtsZ ring is exclusively formed at the midpoint of the cell to initiate the symmetric cell division process [17].

In plants, chloroplast division includes selection of the division site in the middle point of chloroplasts, assembly of the division apparatus and constriction of the chloroplast envelope $[5,18,19]$. Many proteins involved in chloroplast division are homologs of bacterial cell di- 
vision proteins, including FtsZ family, MinD, MinE and ARC6 [6, 9, 20, 21]. FtsZ family of proteins in Arabidopsis includes FtsZ1, FtsZ2 subfamily and ARC3 [20, 22]. They are all important for chloroplast division, but with distinct roles. ARC6 is a homolog of a cyanobacteriaspecific cell division protein, Ftn2, in plants [23]. FtsZ1, FtsZ2 and ARC6 are localized to a ring at the chloroplast division site and are essential components of chloroplast division apparatus [19, 23].

Interestingly, ARC3, although a member of FtsZ family, has a MinCD-like role in determining the division site of chloroplasts [24]. Mutation of $A R C 3$ causes a phenotype of misplacement of chloroplast division site, similar to that of $\min D$ mutant or MinE-overexpressing transgenic plants [22, 25-27]. So far, the homolog of MinC is still missing in higher plants. These data indicate that the division site determining mechanism in chloroplasts in higher plants is somewhat different from that of bacteria.

In this paper, we report the identification of a novel chloroplast division site determinant factor, chloroplast division site positioning 1 (AtCDP1), in Arabidopsis. Like ARC6, AtCDP1 is a homolog of cyanobacteria cell division protein Ftn2. Unexpectedly, $c d p 1$ mutant has a phenotype similar to the AtminD mutant and arc3, but not arc6. We also found that CDP1 interacts with ARC3. Our data suggest that CDP1 has a function different from its homolog ARC6 and represents a new component of the chloroplast division site placement system.

\section{Results}

AtCDP1 affects E. coli cell division and chloroplast division

It was shown before that overexpression of some chloroplast division proteins with prokaryotic origin in $E$. coli can cause a cell division phenotype [28, 29]. Therefore, we constructed an Arabidopsis cDNA expression library in $E$. coli to screen colonies with a cell division phenotype. We found that AtCDP1 (At3g19180), when expressed in E. coli, affected cell division. E. coli cells
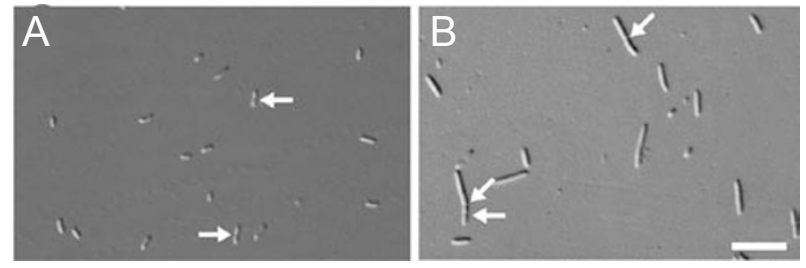

Figure 1 At3g19180 expressed in E. coli affects cell division. (A) DH5 $\alpha$ with $50 \mu \mathrm{M}$ IPTG. (B) Expression of AtCDP1 in DH5 $\alpha$ with $50 \mu \mathrm{M}$ IPTG. Bar= $10 \mu \mathrm{m}$. Arrows mark the cell division site. overexpressing AtCDP1 were heterogeneous and the division site was misplaced (Figure 1A, 1B).

Although two gene structures of AtCDP1 were present in the NCBI database (Figure 2M), only one of them was obtained by screening the expression library (shown with a black asterisk in Figure 2M). Moreover, total Arabidopsis RNA were analyzed by semiquantitative RTPCR with primers at specific locations (Figure 2M, black arrow). Only one type of PCR product was obtained, consistent with one of the gene structures (Figure 2M, marked with a black asterisk; data not shown).

Two T-DNA mutants of $A t C D P 1$ were obtained from ABRC and confirmed by PCR analysis (Supplementary information, Figure S1). One is salk_100009, in which the insertion site is near the $5^{\prime}$ end of the gene and the other line is salk_138043, in which the insertion site is near the $3^{\prime}$ end of the gene (Figure 2M). Both lines showed abnormalities in the size and shape of chloroplasts in mesophyll cells (Figure 2B, 2C), compared to that of wild type (Figure 2A). Especially, there were a few extremely elongated chloroplasts in the bundle sheath cells and multiple constrictions were visible in these enlarged chloroplasts in salk_100009 (Figure 2E). In addition, elongated chloroplasts were also found in the petioles of $c d p 1$ mutants (Figure $2 \mathrm{G}, 2 \mathrm{H}$ ), similar to $\operatorname{arc3}$ mutant (Figure 2I) and arcll mutant (Figure 2J), in which more than one constriction site were formed in elongated chloroplasts. This defective phenotype of chloroplast division was stable during the development in the two T-DNA insertion mutants.

To further confirm the identification of $A t C D P 1$ gene, wild-type AtCDP 1 cDNA was introduced into salk_100009 mutant. The mesophyll cells from transgenic plants were observed and the mutant phenotype was complemented (Figure 2K, 2L). The complementation of T-DNA insertion mutant salk_100009 by wild-type AtCDP1 cDNA indicates that the abnormal chloroplast division phenotype of salk_100009 results from the disruption of $A t C D P 1$ gene.

AtCDP1 is specially expressed in young green tissues in Arabidopsis

To investigate the expression pattern of AtCDP1 gene, a GUS reporter system was used. In transgenic plants, GUS signal was strongly detected in the cotyledon of 3-day-old seedlings (Figure 3A), weakened during the development, and the signal fully disappeared in the cotyledon of 7-day-old seedlings (Figure 3B). The signal was not visible in roots all the time (Figure 3A, 3B). It started to be detected in the shoot apex and the first true leaf of 7-day-old seedlings (Figure 3B) and weakened gradually during the development (Figure 3B top-right). 

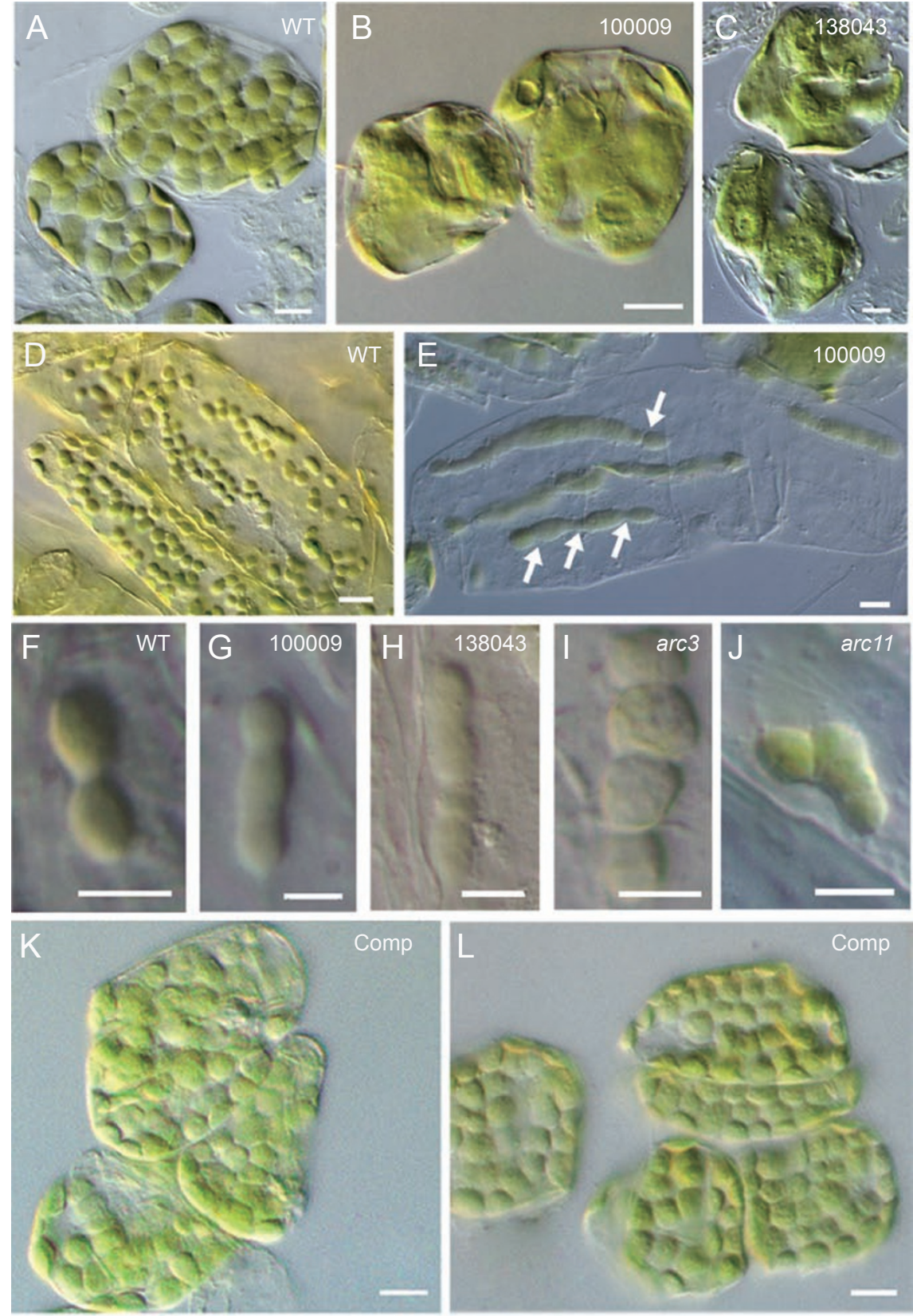

M

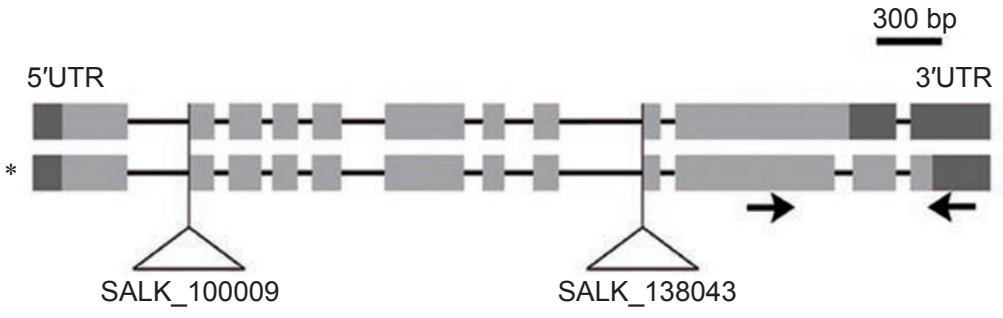

Figure 2 AtCDP1 is a new chloroplast division gene. (A-C) Morphology of chloroplasts in mesophyll cells. (D and E) Chloroplasts in bundle sheath cells. (F-J) Elongated chloroplasts with multiple constriction sites in different mutants. (K and $\mathbf{L}$ ) salk_100009 transformed with the wild-type AtCDP1 cDNA, showing complementation in mesophyll cells based on chloroplast size and number. White arrows mark the constriction site in chloroplasts. All bars are $10 \mu \mathrm{m}$, except in (F) where the bar is $5 \mu \mathrm{m}$. (M) Gene structure of AtCDP1. Exons are depicted as gray rectangles and two T-DNA insertion sites are indicated. A black asterisk indicates the cDNA sequence obtained from screening the cDNA library. bp, base pairs; black arrows show the specific location of primers used in RT-PCR. 


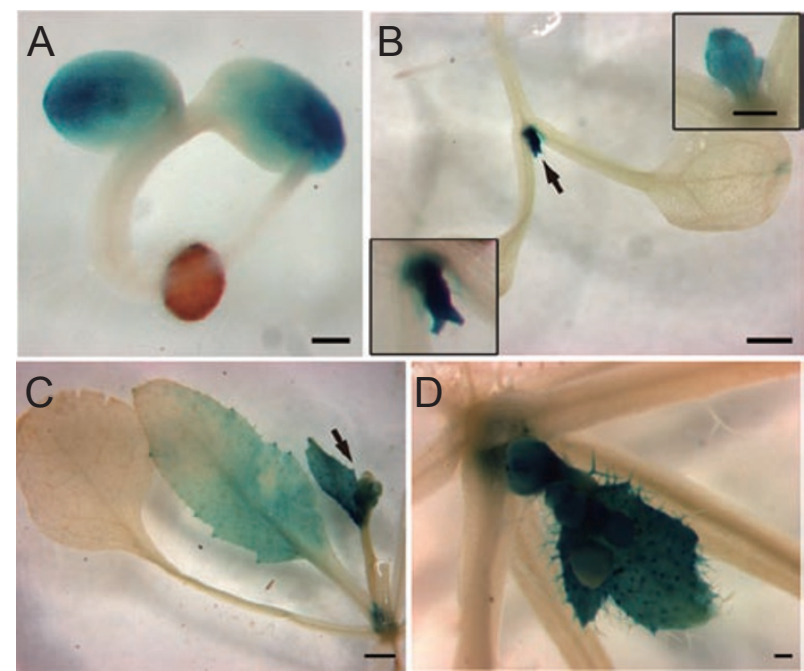

Figure 3 Histochemical GUS staining of $P_{A t C D P 1}$ :GUS transgenic plants. (A) A 4-day-old seedling grown on 1/2 MS medium. (B) Seedlings grown for 7 days on $1 / 2 \mathrm{MS}$ medium. The arrow indicates true leaves, which are enlarged in the inset. (C) Soil grown mature plants. The arrow indicates inflorescence, which is partially enlarged in (D). Bars in A, B and D are $200 \mu \mathrm{m}$, bar in $\mathrm{C}$ is $1 \mathrm{~mm}$.

Generally, the GUS signal was easily detected in young leaves (Figure 3C), and the expression pattern was the younger the leaf, the stronger the signal. In addition, GUS signal was also detected in the budding inflorescence (Figure 3C, 3D), but not in any mature inflorescent tissues (data not shown). After parallel analysis of many transgenic lines with strong expression of GUS, the expression pattern of $A t C D P 1$ was found to have a notable temporal and spatial character in that $A t C D P 1$ was specifically expressed in young green tissues during plant development in Arabidopsis.

\section{AtCDP1 is a chloroplast-targeted protein in Arabidopsis}

$A t C D P 1$ is encoded by a nuclear gene and predicted to have a chloroplast transit peptide (data not shown). To confirm this prediction, the first 90 -amino acid segment was fused to GFP controlled by a CaMV $35 \mathrm{~S}$ promoter and transiently expressed in wild-type Col-0 Arabidopsis protoplasts. Green fluorescence signal was detected in the whole chloroplast, which overlaps with the red color of chlorophyll autofluorescence, indicating that AtCDP1 is associated with chloroplasts (Figure 4D-4F). As a negative control, GFP protein was expressed in wild-type Col-0 Arabidopsis protoplasts. Red chlorophyll autofluorescence could be detected in chloroplasts and the green fluorescence remained in the cytosol (Figure 4A-4C). In the transgenic plants, AtCDP1-GFP was dispersed in the whole chloroplasts both in severe lines (Figure 5B, 5C) and in a mild line (Figure 5D). These results confirmed that AtCDP1 has a functional chloroplast transit peptide and is exclusively targeted to chloroplasts.

Overexpression of AtCDP1 in transgenic plants severely inhibits chloroplast division

To analyze the role of $A t C D P 1$ in chloroplast division, $35 S$-AtCDP1-GFP was stably expressed in transgenic Arabidopsis plants. Transgenic plants were phenotyped at the same developing stage. Some lines had a severe phenotype in chloroplast division (Figure 5B and 5C), some lines had a mild phenotype (Figure 5D) and some lines had no obvious phenotype in the size and number of chloroplasts (data not shown). In transgenic lines with a severe phenotype, mesophyll cells contain only a few enlarged chloroplasts (Figure 5B and 5C), which were clearly distinguishable from that of the wild type (Figure 5A). Furthermore, western blot analysis confirmed that the severe phenotype of chloroplast division was associated with the higher level of AtCDP1 protein in transgenic plants (Figure 5E). Chloroplast division is very sensitive to the level of proteins with prokaryotic origin $[30,31]$. Our results suggest that AtCDP1 plays a key role in chloroplast division process and it may interact with other chloroplast division proteins.

\section{AtCDP1 can self-interact and interact with ARC3}

Chloroplast division requires the assembly of protein complex at the right place in chloroplasts $[5,18$, 19, 32]. To test whether AtCDP1 could form a complex with other known division proteins, protein interaction analyses were performed (Figure 6 and Supplementary

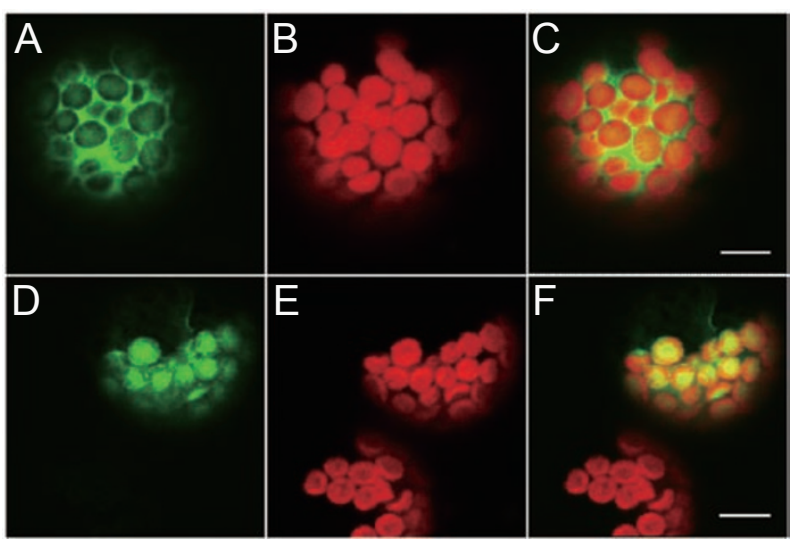

Figure 4 AtCDP1 has a chloroplast transit peptide. (A-C) 35S-GFP transient expression in Arabidopsis protoplasts as a negative control. (D-F) Transient expression of a GFP with an $\mathrm{N}$-terminal fusion of the first 90 aa of AtCDP1 in Arabidopsis protoplasts. GFP, green; chlorophyll autofluorescence, red; Bar $=5 \mu \mathrm{m}$. 


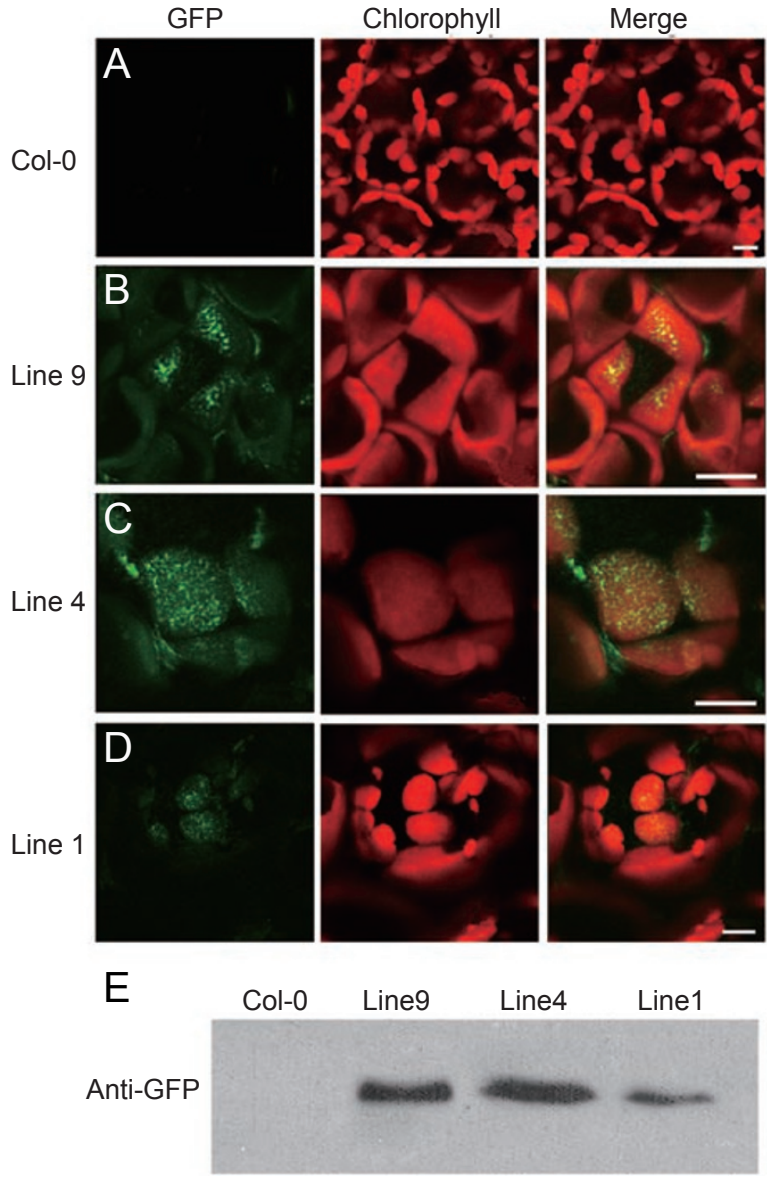

Figure 5 Overexpression of AtCDP1-GFP in wild-type plants blocks chloroplast division. (A) Chloroplast in wild-type Col-0. As a negative control, there is no GFP signal detected. (B-D) Three 35S-AtCDP1-GFP transgenic lines observed by confocal microscopy. Wild-type AtCDP1 was fused with GFP and overexpressed in wild-type Col-0 background. Shown are severe (B and $\mathbf{C}$ ) or mild (D) phenotype in chloroplast size and number. Chlorophyll autofluorescence is red. All samples are collected from 3-day-old seedlings and all bars are $10 \mu \mathrm{m}$. (E) Western blot analysis of the level of AtCDP1-GFP in different transgenic lines by anti-GFP antibodies.

information, Figure S2). First, yeast two-hybrid assay was carried out. AtCDP1 and many known division proteins, such as AtFtsZ1, AtFtsZ2 and ARC3, were fused to the GAL4 activation domain (pGADT7) and GAL4 DNA-binding domain (pGBKT7), respectively. The bait and prey plasmids were co-transformed into the cells of yeast AH109 strain. If the two proteins could interact, the genes for the synthesis of histidine, leucine and tryptophan will be induced and the yeast cells will be able to grow without these amino acids. Because this system is leaky, 3-AT was used to reduce the basal level of growth. The results showed that AtCDP1 could self-interact and also interact with ARC3, but it couldn't interact with AtFtsZ1 or AtFtsZ2 (Figure 6A).

To further confirm the interaction between AtCDP1 and ARC3, we performed a BiFC assay based on the reconstitution of YFP fluorescence when nonfluorescent $\mathrm{N}$-terminal YFP $\left(\mathrm{YFP}^{\mathrm{N}}\right)$ and $\mathrm{C}$-terminal YFP $\left(\mathrm{YFP}^{\mathrm{C}}\right)$ fragments are brought together by two interacting proteins in living plant cells. AtCDP1 and ARC3 were fused to the N-terminal of $\mathrm{YFP}^{\mathrm{N}}$ and the $\mathrm{N}$-terminal of $\mathrm{YFP}^{\mathrm{C}}$,

A

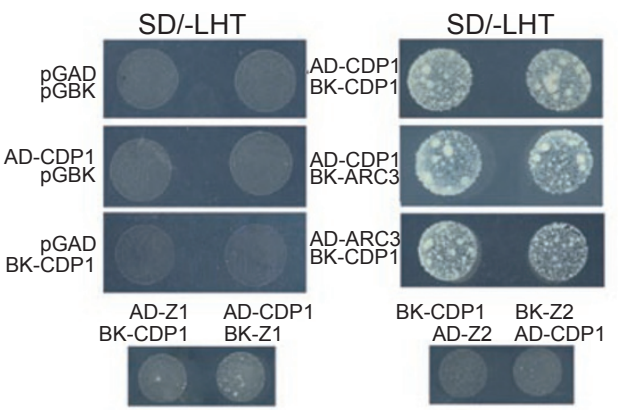

B

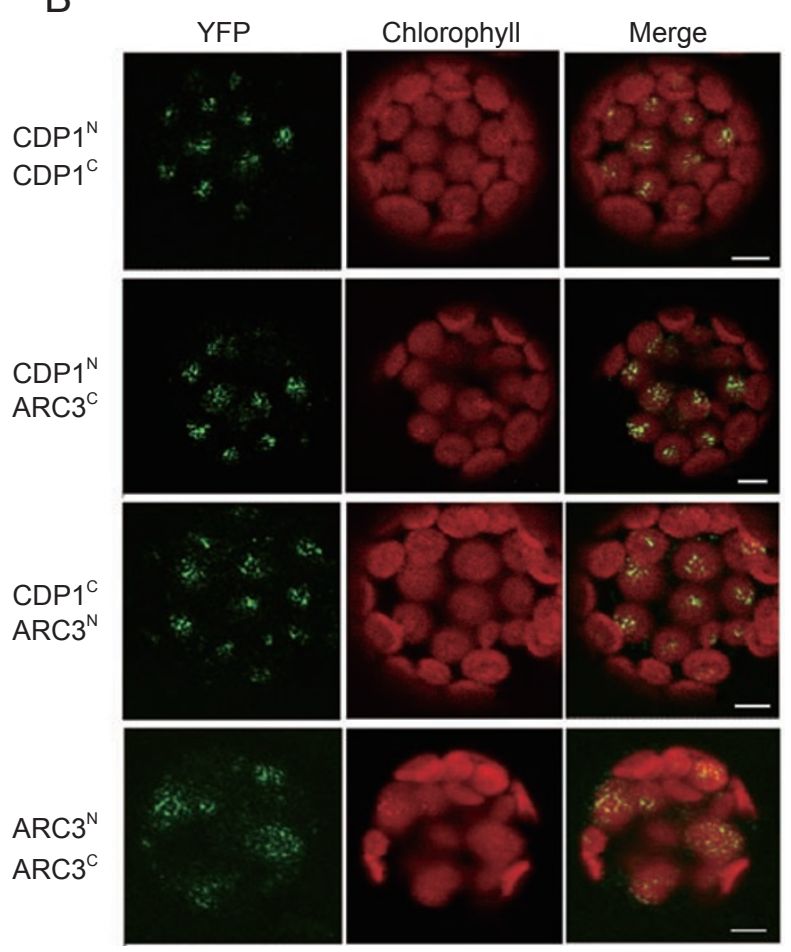

Figure 6 AtCDP1 interacts with ARC3. (A) Yeast two-hybrid analysis. Yeast strain AH109 co-transformed by indicated plasmids were grown on SD medium with $3 \mathrm{mM} 3-\mathrm{AT}$. L, leucine; T, tryptophan; H, histidine; Z1, AtFtZ1-1; Z2, AtFtZ2-1. (B) AtCDP1 interacts with ARC3 and AtCDP1 self-interacts as shown by $\mathrm{BiFC}$ assay in Arabidopsis protoplasts. The YFP signal is represented by green color. CDP $1^{\mathrm{N}}, \mathrm{CDP} 1$ fused with $\mathrm{YFP}^{\mathrm{N}}$; CDP $1^{\mathrm{C}}$, CDP1 fused with YFP ${ }^{\mathrm{C}} . \mathrm{ARC}^{\mathrm{N}}, \mathrm{ARC}$ fused with $\mathrm{YFP}^{\mathrm{N}} ; \mathrm{ARC}^{\mathrm{C}^{C}}$, ARC3 fused with $\mathrm{YFP}^{\mathrm{C}}$. Bar $=5 \mu \mathrm{m}$. 
respectively, and co-transformed into Arabidopsis protoplasts simultaneously. The yellow fluorescent signal was detected in chloroplasts when AtCDP1-YFP ${ }^{\mathrm{N}}$ was cotransformed with ARC3-YFP ${ }^{\mathrm{C}}$ (Figure 6B, the second row) or AtCDP1-YFP ${ }^{\mathrm{C}}$ was co-transformed with ARC3YFP $^{\mathrm{N}}$ (Figure 6B, the third row). These data further demonstrated that AtCDP1 can interact with ARC3 in accordance with the result of the yeast two-hybrid assay (Figure 6A). In addition, the signal of self-interaction was also detected in chloroplasts with co-transformed AtCDP1-YFP ${ }^{\mathrm{N}}$ and AtCDP1-YFP ${ }^{\mathrm{C}}$ in Arabidopsis protoplasts (Figure 6B, the first row). As a positive control, the signal of ARC3 self-interaction was detected in chloroplasts too (Figure 6B, the last row). In negative controls, no YFP signal was detected (Supplementary information, Figure S2).

Both of the above assays suggest that AtCDP1 could not only self-interact, but also interact with ARC3, a chloroplast division protein involved in the division site placement in Arabidopsis.

\section{AtCDP1 is exclusively involved in chloroplast division}

It was shown before that chloroplast ultrastructure and plant development were also affected in some chloroplast division mutants $[33,34]$. So, we investigated the ultrastructure of the chloroplast in salk 100009 and AtCDP1GFP overexpression lines by transmission electron microscopy. Thylakoids in both of them were as normal as that in wild type (Figure 7). In AtCDP1 mutant (Figure 7C, 7D) and transgenic plants (Figure 7E, 7F) with enlarged chloroplasts overexpressing AtCDP1, stroma thylakoids and grana thylakoids were still retained and their ratio was well-balanced as that in wild type. However, if the number of the chloroplasts in one cell in the mutant was less than that in wild type during the same developing stage, the grana were densely packed with more stacks than that of wild type (Figure 7B, 7D). This seems to reflect a compensatory mechanism due to a decrease in the total chloroplast number in one cell. [5]. The plant shape and growth rate of the mutants were almost the same as those of the wild type (data not shown). Either the loss-of-function of AtCDP1 or overexpression of AtCDP1 would lead to imbalance of chloroplast division in Arabidopsis. However, the ultrastructure of chloroplasts and the growth of the plant were not affected (Figure 7 and data not shown). Thus, we propose that AtCDP1 protein is exclusively involved in chloroplast division in Arabidopsis.

\section{Discussion}

Although a dozen of Arabidopsis arc mutants relevant to the chloroplast division process have been reported

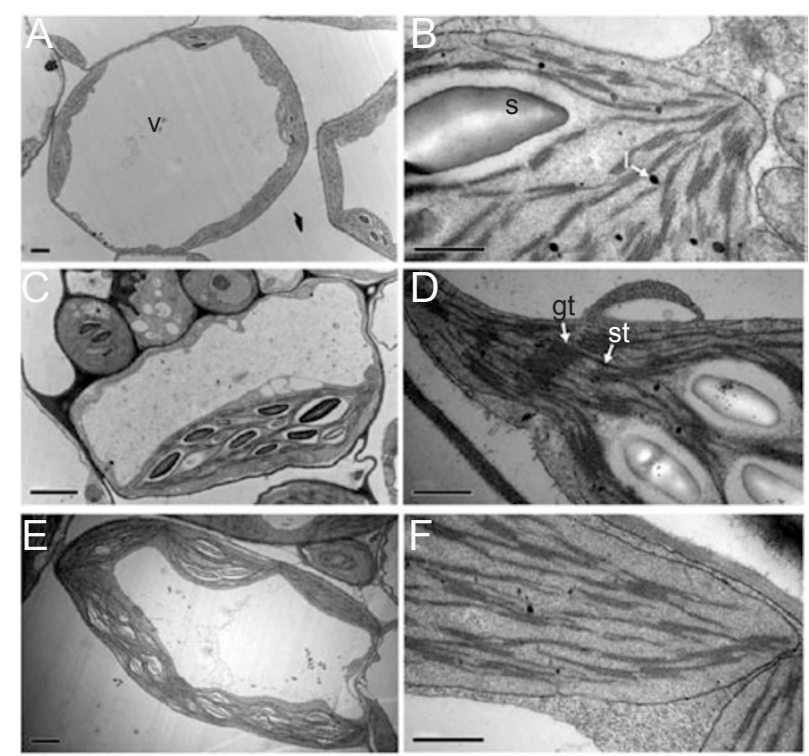

Figure 7 Ultrastructure of chloroplasts. (A and B) Wild-type Col0. (C and D) AtCDP1 mutant Salk_100009. (E and F) Overexpression of AtCDP1-GFP in wild type. gt, grana thylakoids; st, stroma thylakoids; s, starch grain; I, lipid grain; v, vacuole. Bars in $A, C$ and $E$ are $5 \mu \mathrm{m}$, bars in $B, D$ and $F$ are $0.5 \mu \mathrm{m}$.

many years ago $[35,36]$, only a few of them are cloned by map-based cloning during a long period of time [22, 23, 37-39]. Mapping genes for these mutants is laborious, because one has to use microscope for phenotyping $[22,38]$. The function of many chloroplast division proteins with prokaryotic origin is conserved across bacteria and plants. For instance, both AtMinD and AtMinE overexpressed in tobacco plants affected chloroplast division [31, 40]; AtMinE introduced into E. coli led to a minicell phenotype [27] and a chloroplast-targeted $E$. coli MinC overexpressed in tobacco plants also disturbed chloroplast division [41]. Thus, using a bacterial expression system of plant cDNA library may be a new strategy to screen for chloroplast division proteins. Although large numbers of clones need to be examined, it can still reduce the amount of work. Indeed, we found that overexpression of AtCDP1 in E. coli interfered with cell division.

AtCDP1 is evolved from a cyanobacterial cell division protein, Ftn2 [23]. It has a predicted chloroplast transit peptide. By expressing the fusion protein of the predicted chloroplast transit peptide and GFP in Arabidopsis protoplasts, we confirmed that the prediction is true. Moreover, AtCDP1-GFP is targeted to chloroplasts, further suggesting that AtCDP1 is a chloroplast protein.

As expected, T-DNA insertion lines of AtCDP1 have a chloroplast division defect. However, the detailed 
phenotype of $c d p 1$ mutants is surprising. Because both AtCDP1 and ARC6 are derived from Ftn2 [23], it is expected that $c d p 1$ mutant may have a phenotype similar to that of arc6. The arc6 mutant has a chloroplast division defect with only one or two giant chloroplasts [23]. The FtsZ filaments in arc 6 are short fragments, indicating that ARC6 is required for the assembly or stabilization of FtsZ polymer [23]. In $c d p 1$ mutant, chloroplasts are found to be elongated with multiple division sites, which is quite similar to the phenotype of AtminD and $\operatorname{arc} 3$ mutants $[22,26]$. AtMinD and ARC3 function to ensure chloroplast division at the middle point of chloroplasts. In E. coli, MinD and MinC depolymerize FtsZ at nondivision sites [42]. Overexpression of AtMinD in Arabidopsis causes an arc6-like phenotype with short FtsZ filaments and giant chloroplasts [23]. Overexpression of AtCDP1 caused a similar phenotype with blocked chloroplast division. Therefore, CDP1 has an unexpected role similar to that of MinD but contrary to that of its homo$\log$, ARC6.

Although both ARC6 and CDP1 are derived from Ftn2, homologs of these are found in green algae (data not shown), suggesting that they have diverged very early. Their sequences are not very similar, with only $44 \%$ similarity and $21 \%$ identity (data not shown). These data further support that their functions may have diverged too and new function may have been evolved in CDP1.

Yeast two-hybrid and BiFC analyses suggested that AtCDP1 may participate in chloroplast division by interacting with ARC3, which is reported to be a stroma protein and localized to a ring at the midpoint of chloroplasts when the chloroplast division initiates $[5,22,25]$. In addition, ARC 3 has an important role in chloroplast division because it interacts with AtFtsZ1 [25, 32], AtMinD and AtMinE [25] at different locations of chloroplasts as shown by BiFC assay. These data indicate that CDP1 is a new component of the chloroplast division site positioning system.

In E. coli, cell division ring positioning is regulated by MinC, MinD and MinE. These three proteins have a concerted dynamic oscillation from pole to pole in the cell to ensure that the Z-ring forms at the right place [13, 42-45]. Homologs of MinD and MinE were identified in the nuclear genome of Arabidopsis [26, 27, 46], indicating that a Min-like system is a conserved component of the plastid division machinery [9]. But in many aspects, characters of AtMinD and AtMinE are diverged from their homologs in prokaryotes [26, 46, 47]. Recently, it was shown that a plant-specific protein, MCD1, is required for FtsZ ring positioning [48]. MCD1 recruits MinD to the chloroplast inner envelope by direct interaction. The E. coli cell division site positioning system does not include such a protein. Furthermore, oscillation of MinD and MinE in higher plants has not been reported $[12,13]$ and the genuine homolog of MinC has not been found in higher plants, despite that ARC3 is regarded as a MinC-like protein but its function is still not very clear [25]. Now, we show that AtCDP1, as a new component of the Min system, is involved in chloroplast division through the interaction with ARC3 at the early stage of chloroplast division process to ensure the constriction of the ring structure at the midpoint of chloroplasts. All of these data suggest that chloroplast division site positioning has evolved some novel regulatory mechanisms, substituting for the traditional oscillating Min regulation system in bacterial cell division.

\section{Materials and Methods}

\section{Plant material}

The wild-type Columbia (Col-0) ecotype and Salk T-DNA insertion lines Salk_100009 and Salk_138043 for At3g19180 from the ABRC (Ohio State University, Columbus) were used. Surfacesterilized Arabidopsis seeds were sown on 1/2 MS medium or soil and grown under $16 \mathrm{~h} / 8 \mathrm{~h}$ at $22{ }^{\circ} \mathrm{C}$. All transgenic plants were generated by Agrobacterium transformation [49]. Transgenic plants were selected with $50 \mathrm{mg} / \mathrm{L}$ kanamycin or $25 \mathrm{mg} / \mathrm{L}$ hygromycin. To identify T-DNA insertion lines by PCR method (http://signal. salk.edu/cgi-bin/tdnaexpress), primer LBb1 (GCG TGG ACC GCT TGC TGC AAC T) from pBIN-pROK2 was used for all Salk lines. Primers LP9 (TCT CGC ACA TTA GTT ATG GGC) and RP9 (TGC TCA GAA ACT CCG ATA AGC) were used for Salk 100009. Primers LP3 (TTT TCC CAC TGT CTC ACA AGC) and RP3 (TTG TGT ATG CCT GCC GTT AAC) were used for Salk 138043.

\section{Arabidopsis cDNA expression in E. coli}

Total Arabidopsis RNA were extracted with Trizol reagent (Invitrogen, USA) and a cDNA expression library was constructed using SMART cDNA Library Construction Kit (Clontech, USA). All the cDNA sequences were inserted into reconstructive pBluescript SK+ (Stratagene, USA), replacing the sequences between $E c o$ RI and NotI with SfiI A and SfiI B, then transformed into $E$. coli DH5 $\alpha$ and grown in LB medium at $37^{\circ} \mathrm{C}$.

\section{Plasmid construction for transgenic plants}

Full-length $A t C D P 1$ cDNA were PCR-amplified with primer pairs AtCDP1F1: CGC GGATCC ATG CCA GTA GCT TAC ACA TTT C and AtCDP1R1: CGG CTCGAG CTT CTG TAT TTG AAT ATC GC or AtCDP1R2: GGGGATCCT TAC TTC TGT ATT TGA ATA TC and cloned into pGEM-T easy vector (Promega, USA). For the constitutive overexpression vector, GFP was obtained from $p E G F P$ by PCR amplification with primers CGG CTCGAG ATG GTG AGC AAG GGC/CGGAGCTCT TAC TTG TAC AGC TCG TC and introduced between $\mathrm{XhoI}$ and $\mathrm{SacI}$ in pBI121 (Clontech), replacing the $\beta$-glucuronidase sequence with the $G F P$ sequence to generate $p B I-G F P$ vector, and then AtCDP1 was inserted into $p B I$ $G F P$ via $B a m \mathrm{HI} / X h o \mathrm{I}$ to generate $35 S-A t C D P 1-G F P$. To monitor the expression pattern of $A t C D P 1$, a $1.5-\mathrm{kb}$ genomic DNA fragment from the $5^{\prime}$ end of the gene was PCR-amplified using the 
primers CCAAGCTTC TCA GCC TCA TCT T (forward) and CGGGATCCT AAG CTA CTG GCA TTG C (reverse) and fused to uidA reporter gene in the binary plasmid pBI121 (Clontech) between HindIII and BamHI sites.

For complementation analysis, the AtCDP1 ORF was introduced to the BamHI site in pCambia1301 vector. Then the AtCDP1 promoter was cloned into it between HindIII and BamHI sites. $\mathrm{P}_{\mathrm{AtCDP} 1}-\mathrm{AtCDP} 1_{\mathrm{cDNA}}$ was transformed into homozygous AtCDP1 plants. Primers GCA TAA GAG ACC AAT GGA TAC A and CAA ACC TGA GAC TTT CCT ACA C were used for RT-PCR to identify the gene structure.

\section{GUS histochemical staining assay}

The staining protocol was used as previously described [50]. All samples were examined by using Zeiss Stemi SVII (Germany).

\section{Microscopy}

For analyzing the phenotype of chloroplasts, leaves at different stages were collected and prepared as described in [18]. Samples were analyzed with a differential interference contrast microscope (Leica, Oberkochen, Germany). For confocal microscopy, images of chlorophyll autofluorescence and GFP were acquired using a Leica LSM SP2 confocal microscope (Leica, Oberkochen, Germany). Images analysis was done by using Adobe photoshop CS 7.0 software.

\section{Western blot analysis}

Crude proteins were extracted as described in reference [51]. Protein quantification was done by using Bio Rad Dc Protein Assays (Bio-Rad, CA, USA). Total protein (1 mg) was mixed with $5 \times$ sample buffer and separated on a 7.5\% SDS-polyacrylamide gel and transferred to PVDF membrane (Milipore, USA). Immunoblotting was performed with anti-GFP antibodies (G1544, Sigma, USA,). Chemiluminescent detection was conducted by using the Pierce Pico detection reagents (Thermo, USA).

\section{Yeast two-hybrid analysis}

The ORF of AtCDP 1 was PCR-amplified by using primer AtCDP1F2: ATCATATGA TGC CAG TAG CTT ACA CAT TTC$3^{\prime}$ and AtCDP1R2. The ORF of $A R C 3$ was obtained by primers

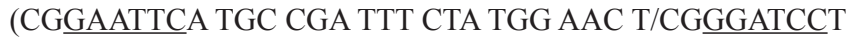
CAA TCT CCG GCG TCC). AtFtsZ1-1 and AtFtsZ2-1 cDNA were amplified using primers pairs (CGGAATTCA TGG CGA TAA TTC CGT TAG/CGGGATCCC TAG AAG AAA AGT CTA CGG GG) and (CGGAATTCA TGG CAA CTT ACG TTT CAC C/CGGGATCCT TAG ACT CGG GGA TAA CGA G). The above genes were cloned into pGADT7 (GAL4 activation domain) and pGBKT7 (GAL4 DNA-binding domain). The constructs, together with empty vector controls (AD and $\mathrm{BD}$ ), were transformed into AH109 yeast cells with different combinations to test the restoration of His auxotrophy with $3 \mathrm{mM} \mathrm{3-AT}$ as recommended by the manufacturer (Matchmaker two-hybrid system 3, Clontech).

\section{Transient expression in Arabidopsis protoplasts}

To test the predicted AtCDP1 transit peptide, the first 90 aa coding sequence was inserted into $p U C 19-M C S-G F P$ [52] in frame with GFP to produce 35S-TPAtCDP1-GFP. For BiFC analysis, the ORF of AtCDP1 was amplified by primers AtCDP1F1/AtCDP1R1 and induced into pUC19-35S-MCS-YFP ${ }^{\mathrm{N}}$ and pUC19-35S-MCS-
$\mathrm{YFP}^{\mathrm{C}}$ [52] via BamHI and XhoI, respectively. The ORF of ARC3 was amplified by primers A3F2, CGGGATCCA TGC CGA TTT CTA TGG AAC and A3R2 CGCTCGAGA TCT CCG GCG TCC ACT TGT, cloned into the vectors above via BamHI and XhoI, respectively. The above constructs were cotransformed into Arabidopsis protoplasts by PEG-mediated method as described previously [53].

\section{Ultrastructure analysis}

The first true leaves from 21-day-old seedlings or 14-day-old seedlings were collected and fixed in $2.5 \%$ glutaraldehyde in PBS overnight at room temperature, washed thoroughly in PBS. For ultrastructural analysis, the first leaf of young Arabidopsis seedlings were fixed, embedded in resin and examined by transmission electron microscopy as previously described for Arabidopsis leaf tissue [54].

\section{Acknowledgment}

We thank the Arabidopsis Biological Resource Center (Ohio State University, Columbus) for providing the Arabidopsis seeds and the editor for careful reading of the manuscript. This work was supported by the National Natural Science Foundation of China (30470879) and Funding Project for Academic Human Resources Development in Institutions of Higher Learning Under the Jurisdiction of Beijing Municipality to He, and National Natural Science Foundation of China (30500288) and Science and Technology Development Program of Beijing Municipal Education Committee grant (KM200610028010) of Beijing Education Committee to $\mathrm{Hu}$.

\section{References}

1 McFadden G. Chloroplast origin and integration. Plant Physiol 2001; 125:50-53.

2 Osteryoung KW, Nunnari J. The division of endosymbiotic organelles. Science 2003; 302:1698-1704.

3 Dyall SD, Brown MT, Johnson PJ. Ancient invasions: from endosymbionts to organelles. Science 2004; 304:253-257.

4 Lopez-Juez E, Pyke KA. Plastids unleashed: their development and their integration in plant development. Int $J$ Dev Biol 2005; 49:557-577.

5 Pyke KA. Plastid division and development. Plant Cell 1999; 11:549-556.

6 Hashimoto H. Plastid division: its origins and evolution. Int Rev Cytol 2003; 222:63-98.

7 Leech RM. Observations on the mechanism of chloroplast division in higher plants. New Phytol 1981; 87: 1-9.

8 Boasson R, Bonner JJ, Laetsch WM. Induction and regulation of chloroplast replication in mature tobacco leaf tissue. Plant Physiol 1972; 49:97-101.

9 Maple J, Moller SG. Plastid division: evolution, mechanism and complexity. Ann Bot (Lond) 2007; 99:565-579.

10 Bi E, Lutkenhaus J. FtsZ regulates frequency of cell division in Escherichia coli. J Bacteriol 1990; 172:2765-2768.

11 Rothfield L, Justice S, Garcia-Lara J. Bacterial cell division. Annu Rev Genet 1999; 33:423-448.

$12 \mathrm{Yu} \mathrm{XC}$, Margolin W. FtsZ ring clusters in min and partition mutants: role of both the Min system and the nucleoid in reg- 
ulating FtsZ ring localization. Mol Microbiol 1999; 32:315326.

13 de Boer PA, Crossley RE, Rothfield LI. A division inhibitor and a topological specificity factor coded for by the minicell locus determine proper placement of the division septum in E. coli. Cell 1989; 56:641-649.

14 de Boer PA, Crossley RE, Hand AR, Rothfield LI. The MinD protein is a membrane ATPase required for the correct placement of the Escherichia coli division site. EMBO J 1991; 10:4371-4380.

$15 \mathrm{Hu}$ Z, Lutkenhaus J. Topological regulation of cell division in Escherichia coli involves rapid pole to pole oscillation of the division inhibitor MinC under the control of MinD and MinE. Mol Microbiol 1999; 34:82-90.

$16 \mathrm{Fu} \mathrm{X}$, Shih YL, Zhang Y, Rothfield LI. The MinE ring required for proper placement of the division site is a mobile structure that changes its cellular location during the Escherichia coli division cycle. Proc Natl Acad Sci USA 2001; 98:980-985.

17 Gitai Z, Shapiro L. Bacterial cell division spirals into control. Proc Natl Acad Sci USA 2003; 100:7423-7424.

18 Miyagishima SY, Froehlich JE, Osteryoung KW. PDV1 and PDV2 mediate recruitment of the dynamin-related protein ARC5 to the plastid division site. Plant Cell 2006; 18:25172530 .

19 Vitha S, McAndrew RS, Osteryoung KW. FtsZ ring formation at the chloroplast division site in plants. J Cell Biol 2001; 153:111-120.

20 Osteryoung KW, Vierling E. Conserved cell and organelle division. Nature 1995; 376:473-474.

21 Osteryoung KW, Pyke KA. Plastid division: evidence for a prokaryotically derived mechanism. Curr Opin Plant Biol 1998; 1:475-479.

22 Shimada H, Koizumi M, Kuroki K, et al. ARC3, a chloroplast division factor, is a chimera of prokaryotic FtsZ and part of eukaryotic phosphatidylinositol-4-phosphate 5-kinase. Plant Cell Physiol 2004; 45:960-967.

23 Vitha S, Froehlich JE, Koksharova O, Pyke KA, van Erp H, Osteryoung KW. ARC6 is a J-domain plastid division protein and an evolutionary descendant of the cyanobacterial cell division protein Ftn2. Plant Cell 2003; 15:1918-1933.

24 Pyke KA, Leech RM. A genetic analysis of chloroplast division and expansion in Arabidopsis thaliana. Plant Physiol 1994; 104:201-207.

25 Maple J, Vojta L, Soll J, Moller SG. ARC3 is a stromal Z-ring accessory protein essential for plastid division. EMBO Rep 2007; 8:293-299.

26 Colletti KS, Tattersall EA, Pyke KA, Froelich JE, Stokes KD, Osteryoung KW. A homologue of the bacterial cell division site-determining factor MinD mediates placement of the chloroplast division apparatus. Curr Biol 2000; 10:507-516.

27 Maple J, Chua NH, Moller SG. The topological specificity factor AtMinE1 is essential for correct plastid division site placement in Arabidopsis. Plant J 2002; 31:269-277.

28 Maple J, Moller SG. Interdependency of formation and localisation of the Min complex controls symmetric plastid division. J Cell Sci 2007; 120:3446-3456.

$29 \mathrm{Hu}$ Y, Kong DD, Wang D, He YK. [Cloning and expression in E.coli of Chlamydomonas reinhardtii CrFtsZ3 gene]. Sheng
Wu Hua Xue Yu Sheng Wu Wu Li Xue Bao (Shanghai) 2003; 35:998-1004.

30 Osteryoung KW, Stokes KD, Rutherford SM, Percival AL, Lee WY. Chloroplast division in higher plants requires members of two functionally divergent gene families with homology to bacterial ftsZ. Plant Cell 1998; 10:1991-2004.

31 Reddy MS, Dinkins R, Collins GB. Overexpression of the Arabidopsis thaliana MinE1 bacterial division inhibitor homologue gene alters chloroplast size and morphology in transgenic Arabidopsis and tobacco plants. Planta 2002; 215:167176.

32 McAndrew RS, Olson BJ, Kadirjan-Kalbach DK, et al. In vivo quantitative relationship between plastid division proteins FtsZ1 and FtsZ2 and identification of ARC6 and ARC3 in a native FtsZ complex. Biochem J 2008; 412:367-378.

33 Robertson EJ, Pyke KA, Leech RM. Arc6, an extreme chloroplast division mutant of Arabidopsis also alters proplastid proliferation and morphology in shoot and root apices. $J$ Cell Sci 1995; 108 (Pt 9):2937-2944.

34 Gao H, Sage TL, Osteryoung KW. FZL, an FZO-like protein in plants, is a determinant of thylakoid and chloroplast morphology. Proc Natl Acad Sci USA 2006; 103:6759-6764.

35 Pyke KA, Leech RM. Rapid image analysis screening procedure for identifying chloroplast number mutants in mesophyll cells of Arabidopsis thaliana (L.) Heynh. Plant Physiol 1991; 96:1193-1195.

36 Pyke KA, Leech RM. Chloroplast division and expansion is radically altered by nuclear mutations in Arabidopsis thaliana. Plant Physiol 1992; 99:1005-1008.

37 Marrison JL, Rutherford SM, Robertson EJ, Lister C, Dean C, Leech RM. The distinctive roles of five different ARC genes in the chloroplast division process in Arabidopsis. Plant $J$ 1999; 18:651-662.

38 Gao H, Kadirjan-Kalbach D, Froehlich JE, Osteryoung KW. ARC5, a cytosolic dynamin-like protein from plants, is part of the chloroplast division machinery. Proc Natl Acad Sci USA 2003; 100:4328-4333.

39 Fujiwara MT, Nakamura A, Itoh R, Shimada Y, Yoshida S, Moller SG. Chloroplast division site placement requires dimerization of the ARC11/AtMinD1 protein in Arabidopsis. $J$ Cell Sci 2004; 117:2399-2410.

40 Dinkins R, Reddy MS, Leng M, Collins GB. Overexpression of the Arabidopsis thaliana MinD1 gene alters chloroplast size and number in transgenic tobacco plants. Planta 2001; 214:180-188.

41 Tavva VS, Collins GB, Dinkins RD. Targeted overexpression of the Escherichia coli MinC protein in higher plants results in abnormal chloroplasts. Plant Cell Rep 2006; 25:341-348.

42 Raskin DM, de Boer PA. The MinE ring: an FtsZ-independent cell structure required for selection of the correct division site in E. coli. Cell 1997; 91:685-694.

43 Raskin DM, de Boer PA. Rapid pole-to-pole oscillation of a protein required for directing division to the middle of $E S$ cherichia coli. Proc Natl Acad Sci USA 1999; 96:4971-4976.

44 Rowland SL, Fu X, Sayed MA, Zhang Y, Cook WR, Rothfield LI. Membrane redistribution of the Escherichia coli MinD protein induced by MinE. J Bacteriol 2000; 182:613-619.

45 Hale CA, Meinhardt H, de Boer PA. Dynamic localization cycle of the cell division regulator MinE in Escherichia coli. 
EMBO J 2001; 20:1563-1572.

46 Itoh R, Fujiwara M, Nagata N, Yoshida S. A chloroplast protein homologous to the eubacterial topological specificity factor minE plays a role in chloroplast division. Plant Physiol 2001; 127:1644-1655.

47 Aldridge C, Moller SG. The plastid division protein AtMinD1 is a Ca2+-ATPase stimulated by AtMinE1. J Biol Chem 2005; 280:31673-31678.

48 Nakanishi H, Suzuki K, Kabeya Y, Miyagishima SY. Plantspecific protein MCD1 determines the site of chloroplast division in concert with bacteria-derived MinD. Curr Biol 2009; 19:151-156.

49 Clough SJ, Bent AF. Floral dip: a simplified method for Agrobacterium-mediated transformation of Arabidopsis thaliana. Plant J 1998; 16:735-743.

50 Nakajima K, Furutani I, Tachimoto H, Matsubara H, Hashi- moto T. SPIRAL1 encodes a plant-specific microtubulelocalized protein required for directional control of rapidly expanding Arabidopsis cells. Plant Cell 2004; 16:1178-1190.

51 Martinez-Garcia JF, Monte E, Quail PH. A simple, rapid and quantitative method for preparing Arabidopsis protein extracts for immunoblot analysis. Plant J 1999; 20:251-257.

52 Zhang M, Hu Y, Jia J, Gao H, He Y. A plant MinD homologue rescues Escherichia coli HL1 mutant (Delta MinDE) in the absence of MinE. BMC Microbiol 2009; 9:101.

53 Yoo SD, Cho YH, Sheen J. Arabidopsis mesophyll protoplasts: a versatile cell system for transient gene expression analysis. Nat Protoc 2007; 2:1565-1572.

54 Pyke KA, Rutherford SM, Robertson EJ, Leech RM. Arc6, a fertile Arabidopsis mutant with only two mesophyll cell chloroplasts. Plant Physiol 1994; 106:1169-1177.

(Supplementary information is linked to the online version of the paper on the Cell Research website.) 